\title{
THE CHARACTERISTICS AND SIMILARITY OF PRIMARY AND SECONDARY PERISTALSIS IN THE ESOPHAGUS *
}

\author{
By BERTRAM FLESHLER, $\dagger$ THOMAS R. HENDRIX, PHILIP KRAMER, AND \\ FRANZ J. INGELFINGER
}

\author{
(From the Evans Memorial, Massachusetts Memorial Hospitals and Department of Medicine, \\ Boston University School of Medicine, Boston, Mass.)
}

(Submitted for publication August 5, 1958; accepted September 11, 1958)

Primary and secondary peristalsis in the esophagus are believed to differ in their modes of initiation and propagation. Since primary peristalsis is, by definition, initiated by a swallow and secondary peristalsis set off by esophageal distention, their difference with respect to initiation is an $a$ priori matter. Recent studies $(1,2)$, however, challenge the concept that a clear distinction exists as far as propagation is concerned.

Classically, primary peristalsis is said to be centrally integrated-that is, the afferent impulse released by swallowing stimulates a medullary center which thereupon, without further afferent stimulation, activates in succession oral, pharyngeal and esophageal musculature (3). Secondary peristalsis was contrasted by Meltzer and Auer (4) as follows: "This peristaltic movement, however, differs from the normal peristalsis after deglutition in the essential point that it does not progress in the lower section of the oesophagus if the latter be divided transversely at some place. That shows that this form of peristalsis depends upon consecutive stimulations of the mucous membrane of the oesophagus and consists of a chain of local reflexes."

The nature of secondary peristalsis has generally been investigated by inserting a bolus into the esophagus and analyzing the transport phenomena elicited. This technique, however, has obvious limitations since the presense of a bolus could elicit local stimuli and reflexes on moving downstream. Under these circumstances, it is impossible to tell whether secondary peristalsis, once elicited, would similarly pass down the

* Presented in part at the meeting of the Gastroenterology Research Group, Colorado Springs, Colo., May 23, 1957.

† Work performed during period as Public Health Service Research Fellow of the National Cancer Institute. Present address: Department of Medicine, Cleveland Metropolitan General Hospital, Cleveland 9, Ohio. esophagus were no bolus present. With modern manometric devices this question can now be solved, for the propagation of secondary peristalsis can be analyzed without using liquid, semisolid or solid boluses that might stimulate local reflexes. We have therefore initiated secondary peristalsis in the human esophagus by local distention, have studied the resultant inhibitory and motor phenomena by the technique of intraluminal manometry, and have compared the effects produced with those obtained when primary peristalsis is induced by swallowing.

\section{METHODS}

A tube assembly, as used in previous studies (5) and consisting of three open-tipped and water-filled polyvinyl tubes, was passed via the nose into the esophagus of healthy volunteers. The tips were spaced $3 \mathrm{~cm}$. apart. Pressure changes at the tips were transmitted through the water columns to Sanborn electromanometers and recorded. An external pneumograph identified respiratory excursions on one channel. All recordings of intraluminal esophageal pressure were made with the subject supine and the manometers leveled at the posterior axillary line. The high pressure zone which is found in the distal esophagus and ascribed to the lower esophageal sphincter (6) was located by first placing the distal recording tip in the stomach and then withdrawing it centimeter by centimeter.

A balloon of $40 \mathrm{ml}$. capacity was attached to a Levin tube; passed into the esophagus, and the proximal end of the tube firmly fixed. Radiologic localization of the balloon and of the radiopaque pressure recording tips was carried out in all cases. The balloon was inflated by several techniques. Momentary or prolonged inflation was carried out by injecting an absolute amount of air rapidly with a syringe, holding the air in the balloon for the desired period of time, and then aspirating the air quickly. To achieve a more physiologic distention stimulus which would not move but would yield to esophageal contraction isobaric inflation of the balloon was used. In this system, which has been used extensively to record motility $(7,8)$, the balloon is inflated under moderate, approximately constant pressure (6 to $12 \mathrm{~cm}$. water) produced by the difference between water levels of two 
chambers in a damped kymographic system (9). The amount of air allowed in the balloon is determined by the esophageal reaction: Once the balloon has reached a certain size, the esophagus contracts and expels air back into the external system. During the various techniques of inflation used, the balloon was also connected to an electromanometer and writing channel in order to provide for accurate recording of times of inflation and deflation, or of esophageal contraction or relaxation in the area of the balloon.

\section{RESULTS}

\section{Effect of momentary distention on esophageal motility}

Rapid inflation and deflation of the fixed balloon was carried out 115 times in 11 subjects. The balloon was held inflated for a mean time of 3.7 seconds. In 55 tests ( 47 per cent), progressive pressure waves were produced and recorded as passing sequentially over tips placed in the esophagus distal to the ballon (Figure 1A). No swallowing movements preceded the appearance of these waves. A nonprogressive pres- sure change appearing in only one tip, called a "spasm," was elicited in an additional seven tests. The amount of air used for inflation of the balloon varied from 5 to $40 \mathrm{ml}$. with a mean of $21 \mathrm{ml}$. No correlation was found between the amounts of air used and the incidence of either progressive or nonprogressive positive pressure responses. Substernal pain was occasionally produced when 35 to $40 \mathrm{ml}$. was injected but did not appear to affect the results. In those tests in which a moving pressure wave was elicited by esophageal distention, the interval between beginning of balloon inflation and the start of the pressure rise at the tip nearest the balloon was 5.5 seconds; the interval between the beginning of balloon deflation and the start of pressure rise at the nearest tip, 2.1 seconds. Since the duration of inflation averaged 3.7 seconds, it appears that initiation of the moving pressure wave in this experimental design was closely related to deflation. The speed of progression of the wave, once set off, averaged $3 \mathrm{~cm}$. per second.
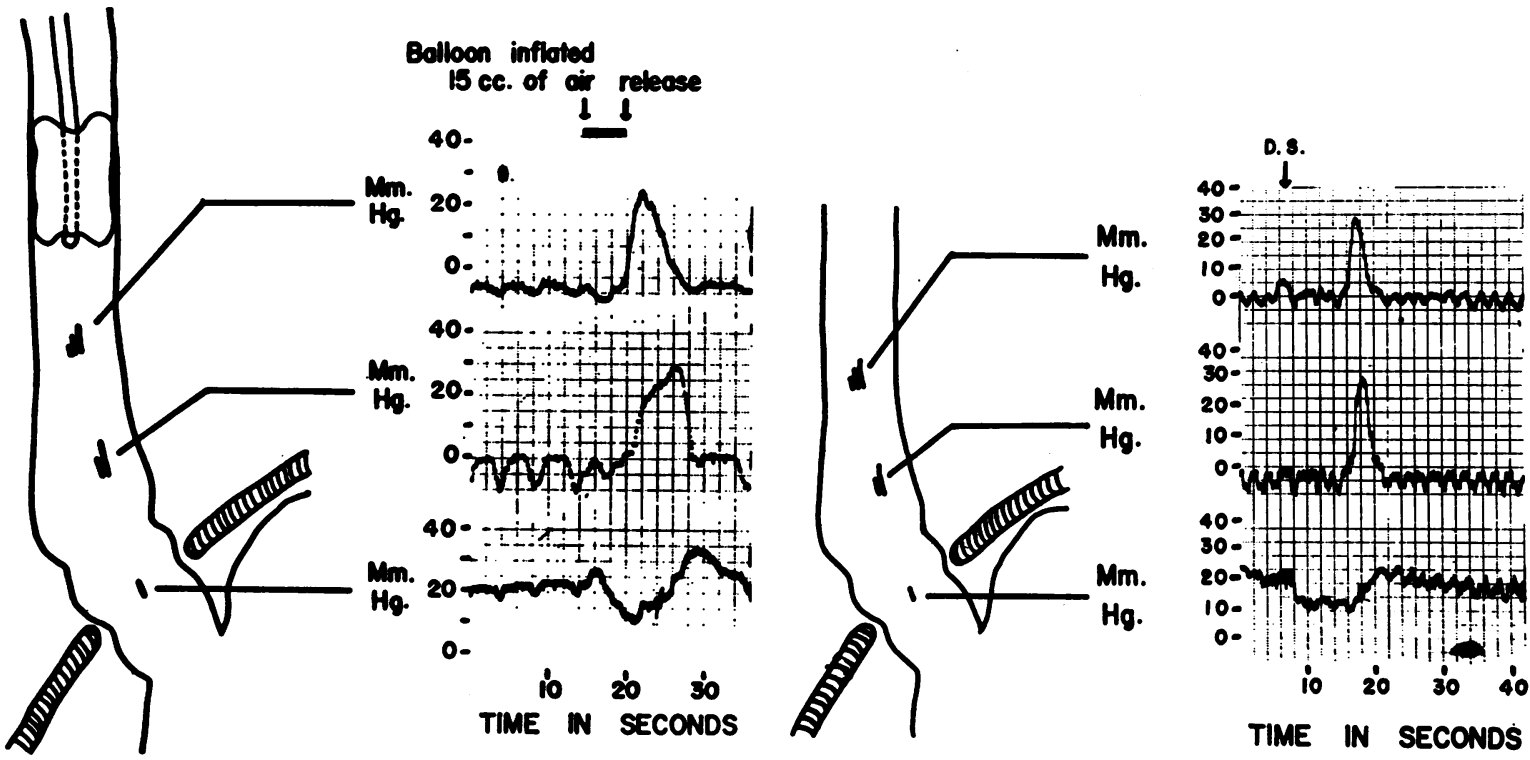

Fig. 1. Comparison of Secondary (A) and Primary (B) Esophagral Peristalsis Curves

A. Momentary distention with an air-filled balloon fixed in the mid-esophagus produced a drop in pressure in the sphincteric zone (bottom tracing), followed by a progressive contraction recorded at two esophageal sites distal to the balloon (upper two tracings). These pressure phenomena, elicited without a swallow or moving bolus, are comparable to those characterizing primary esophageal peristalsis initiated by swallowing (Fig. 1B).

Reprinted with permission from The Journal of Applied Physiology, 1958, 12, 341.

B. The drop in pressure in the sphincteric zone (bottom tracing) and the progressive contraction in the esophagus (upper two tracings) released by a dry swallow (one containing only air and a little saliva) are demonstrated. DS indicates dry swallow. 


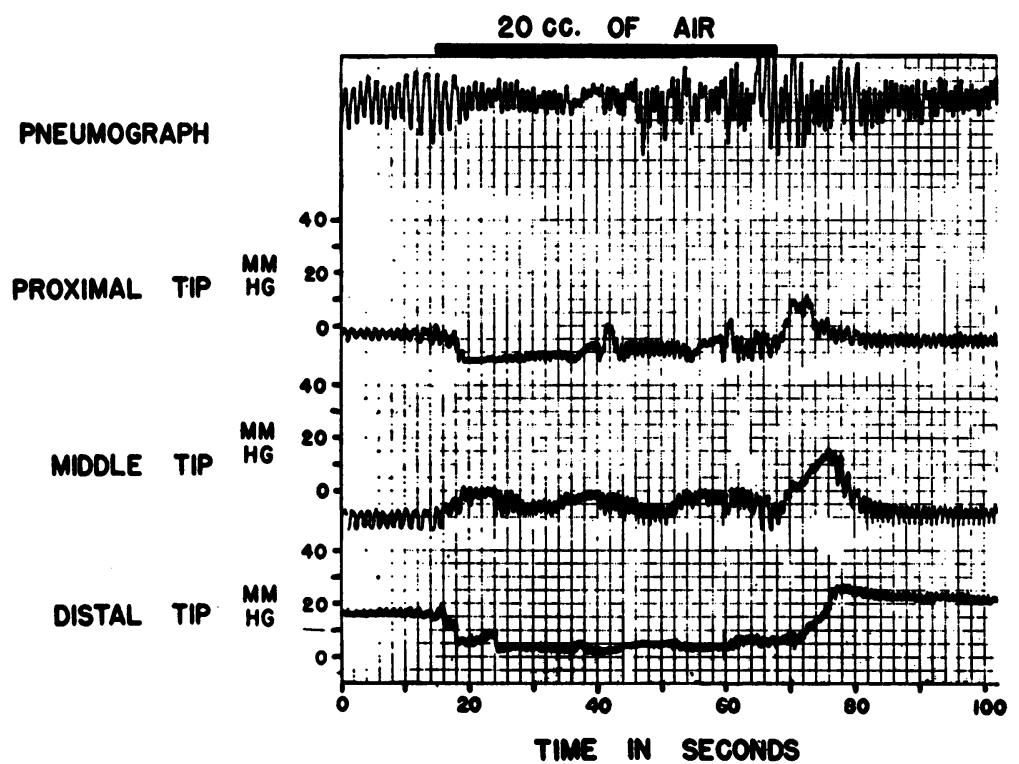

PNEUMOGRAPH

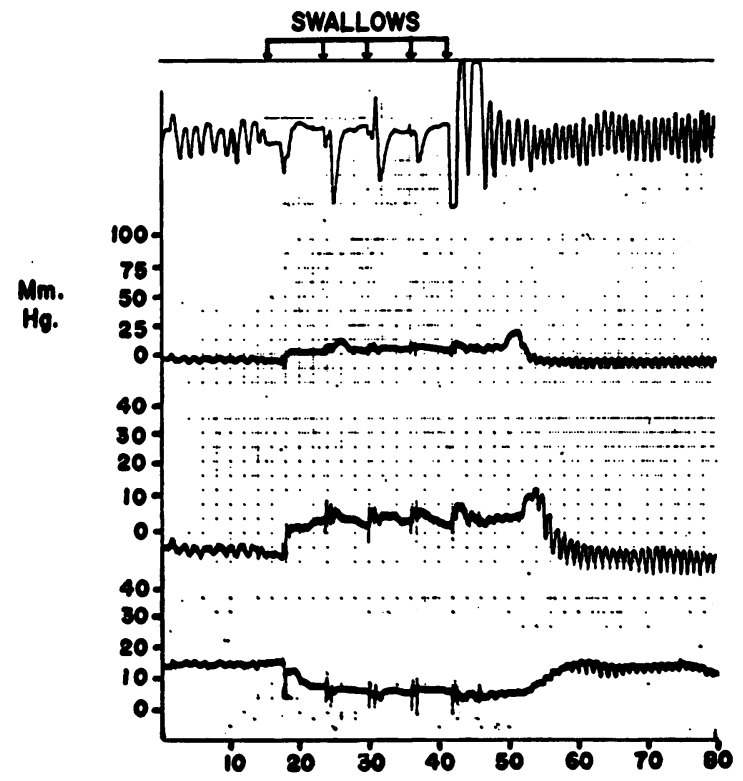

TIME IN SECONDS

Fig. 2. Pressure Tracings During Prolonged Distention (A) Compared with Frequent Swallowings (B)

A. Prolonged distention (solid bar at top of record) of a balloon fixed in the mid-esophagus was attended by no changes in the body of the esophagus distal to the balloon (proximal and middle tips), and produced a sustained drop in intrasphincteric pressure (distal tip), maintained until the balloon was deflated. Thereafter a pressure wave passed through the lower esophagus and sphincter. The overall effect on esophageal motility is similar to that produced by rapidly repeated swallows (Fig. 2B).

B. Liquid swallows taken at approximately seven second intervals produced a sustained drop in pressure in the sphincteric zone (distal tip), and no peristaltic pressure phenomena appeared in the body of the esophagus (proximal and middle tips) during this time. The sustained rise and the occasional spikes of pressure recorded are ascribable to fluid introduced into the esophagus and to transmitted oropharyngeal pressures. After the swallowing efforts ceased, a peristaltic contraction travelled down the esophagus. 
2. Effect of momentary distention on the lower esophageal sphincter

The lowermost recording tip was in the high pressure zone (lower esophageal sphincter) on 62 of the occasions when the balloon was inflated momentarily. A drop in pressure occurred 51 times, 39 being associated with the progressive pressure reactions described above. In every instance the drop in pressure in the sphincteric area preceded the rise in pressure recorded by the tip nearest the balloon (Figure 1A). The average time between the beginning of inflation and the beginning of the pressure drop was 1.5 seconds. The average time from the start of the pressure drop to deflation was 2.2 seconds. Thus the drop in pressure in the sphincteric zone seems to be related to the period of inflation when the esophagus is still distended. At the end of the period of low intrasphincteric pressure, an overshoot to levels 2 to $7 \mathrm{~mm}$. Hg above resting levels occurred in 12 of the 39 tests in which progressive esophageal pressure waves were elicited by momentary distention.

In 12 instances a pressure drop in the sphincter zone occurred in the absence of any accompanying progressive pressure wave. The average amount of air used for ballon inflation in these 12 tests was $17 \mathrm{ml}$. as compared to the average amount of $21 \mathrm{ml}$. used to elicit the full sequence of an esophageal progressive pressure wave and a decrease in intrasphincteric pressure. In the group of 12 tests exhibiting only a drop in intrasphincteric pressure, this drop was less marked than in those exhibiting the full sequence, and in no case did pressures overshoot the resting levels at the completion of the low pressure interval.

No evidence of sphincteric changes was detected in the seven tests showing a "spasm" response.

\section{Effects of prolonged distention}

When inflation was maintained for periods of 10 seconds to 2 minutes, with no air allowed to escape from the balloon, variable patterns resulted. One effect was a drop in pressure in the high pressure zone which continued for as long as the balloon was inflated (Figure 2A). During this period no clear-cut pattern of pressure changes occurred in the body of the esophagus. After deflation, a progressive pressure wave was recorded passing over the esophagus and the sphincteric area. In this case, as was true of the response to momentary distention, the pressure drop in the high pressure zone seemed related to inflation, whereas the positive pressure sequence in the body of the esophagus seemed to be related to balloon deflation.

Prolonged distention sometimes produced no changes in the motor activity of either the esopha-

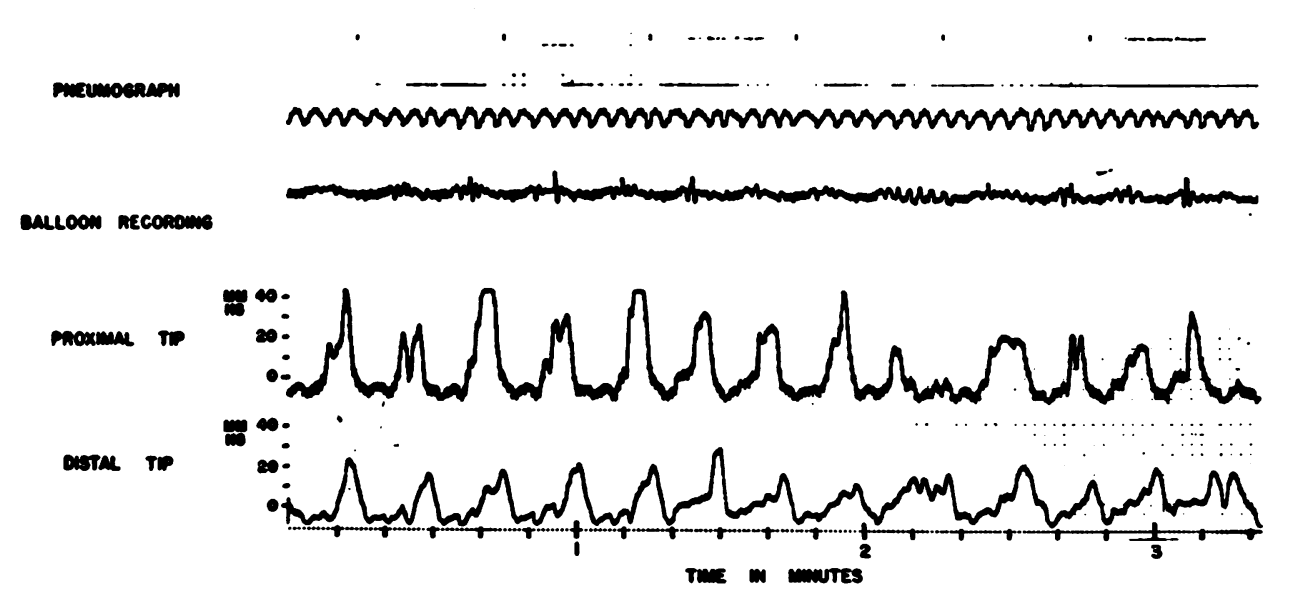

Fig. 3. Effect of Isobaric Distention on Esophageal Contractions

Balloon inflation by the isobaric technique produced repeated local esophageal contractions (small spikes in balloon recording). Each contraction compressing the fixed but pliable balloon bolus was followed by a pressure wave progressing through the body of the esophagus below the balloon (proximal and distal tips). 
gus or the sphincteric area. On occassion, a pressure wave was initiated and progressed down the esophagus while prolonged distention was being maintained. In these instances, the response in the esophagus distal to the balloon appeared to be preceded by a fall and then a rise of pressure within the balloon, as if the resistance of the esophageal wall to balloon distention had to diminish before a distally moving pressure wave could be elicited.

\section{Effect of isobaric distention}

When the esophagus in five subjects was stimulated by isobaric balloon inflation, air flowed into the balloon under the applied pressure and then flowed out in response to the resultant esophageal contraction at a rate of four to nine full cycles per minute. Each of the contractions so produced by the fixed but pliable balloon stimulus thereupon travelled down the gullet at a corresponding rate of four to nine per minute (Figure 3 ) and produced a moving pressure complex similar to those seen in response to momentary balloon distention. Diaphragmatic contraction, as reflected in the pneumograph, could not be correlated with the moving pressure waves.

In three cases in which the distal recording tip was in the lower esophageal sphincter zone, symmetrically spaced periods of high and low pressure were also recorded. Though not always identifiable against this fluctuating background, intrasphincteric pressure seemed to fall shortly after the beginning of each contraction about the stimulating balloon.

\section{Pressure patterns produced in the esophagus above the balloon}

Pressures were recorded at three sites (spaced $3 \mathrm{~cm}$. apart) above the balloon during and after 21 momentary inflations in three subjects. A simultaneous increase in pressure occurred in 19 cases at two or more recording sites. In four of these, a progressive pressure wave followed the "spasm." These pressure waves had the same configuration and rate of progress as those recorded in the esophagus below a stimulating balloon. In no instance was a progressive pressure wave seen above the balloon without an antecedent "spasm."

Isobaric distention, with recording above the balloon, resulted in a different pattern from that obtained below the balloon. Instead of repetitive contractions moving aborally at a rate of four to nine per minute, "spasms," usually erratic but occasionally rhythmic at a rate of approximately nine per minute, were recorded.

\section{DISCUSSION}

The aborally moving pressure wave produced by momentary local distention of the mid-esophagus exhibits a pattern identical to that released by a "dry" swallow (Figure 1B). The configuration of the complex, its rate of progression, and the fall in pressure in the zone of the lower esophageal sphincter are common to both motor phenomena, although one is the result of a swallow, i.e., primary peristalsis, and the other of local esophageal distention, i.e., secondary peristalsis. No swallowing, moving bolus or respiratory effects attended the secondary wave. Thus local esophageal distention may elicit a peristaltic phenomenon in the esophagus exactly the same as that of primary peristalsis, except that manifestations of a nervous control center, such as oropharyngeal movements and respiratory interruption, are absent. Since no bolus moved downstream, propagation of the wave obviously did not depend on a bolus to set off a series of "consecutive stimulations of the mucous membrane of the oesophagus."

Although a regular pattern of secondary peristalsis could be elicited by momentary distention, the response was obtained in no more than half the tests. Creamer and Schlegel, in studying the esophageal response to distention, observed peristalsis distal to the distending balloon in only 26 per cent of tests (2). Even swallowing, however, is irregularly followed by a primary peristaltic wave, the response occurring about three-fourths of the time in the entire esophagus, and 59 per cent of the time in the lower third (10). In the distention experiments, one reason for a peristaltic response rate may lie in the stimulus itself. Certainly forcible inflation of a balloon is not comparable as a stimulus to a swallow of water and may, indeed, favor a response of inhibition over contraction.

Similar and powerful inhibitory influences participating in the control of esophageal motility 
may be brought to light both by swallowing and by local esophageal distention. Either stimulus may be followed by a fall in pressure in the zone of the lower esophageal sphincter, presumably reflecting relaxation of tonically contracted muscle. As evident from the results of momentary esophageal distention, this sphincteric relaxation precedes the onset of peristaltic contraction in the body of the esophagus and may, indeed, take place without a detectable contractile response, an observation also made by others in man (2) and dogs (11). When the inhibitory phase is constantly stimulated, either by taking repetitive swallows at intervals of less than seven seconds (Figure 2B) or by maintaining forcible local distention of the esophagus, the sphincteric zone remains constantly relaxed.

Inhibitory influences in the sphincteric zone are readily demonstrable because of its high resting tonicity. In the body of the esophagus, flaccid at rest, inhibitory effects are not so easily shown but their presence may be inferred. It is evident that repetitive swallows at less than seven second intervals or prolonged balloon distention will postpone until after cessation of the stimulus the peristaltic contraction that follows a single swallow or momentary inflation of the balloon. Thus, stimuli eliciting either primary or secondary peristalsis would seem to release first a wave of inhibition that passes rapidly down the esophagus and the lower sphincter; when this phase ends, and only then, a contractile effect travels the same path. The "wave of inhibition" need not be regarded as a wave of positive inhibitory influences. It is more simply conceived of as a temporary suppression of excitatory stimuli, phasic as well as tonic.

The mere release of an inhibitory effect does not necessarily precipitate an ensuing contraction. Rather, a local contraction appears either if a rigid distending force is released, or if a moderate and yielding distending force is employed, as is the case if a liquid bolus is introduced into the esophagus or isobaric balloon distention is used. Propagation of this contraction in the form of a peristaltic wave then takes place, possibly because sensory stimuli from the area of local contraction set off a chain reaction in some nervous swallowing center controlling esophageal peristalsis. If this were the case, however, the esophageal motor response above the stimulated area might be expected also to exhibit a peristaltic pattern. Such a pattern was not recorded at all during isobaric distention of the esophagus, and after momentary distention only if a "spasm" was first elicited. A central nervous mechanism organizing secondary peristalsis should also act less erratically with respect to the incidence of peristaltic phenomena appearing in response to local esophageal stimulation. Certainly the nervous center that controls oropharyngeal swallowing mechanisms is distinguished by the constancy and reproducibility of its sequence (12).

The alternate possibility is that the local stimulus sets off a contractile impulse which passes down the esophagus independently of a swallowing center. Responsible pathways would include vago-vagal reflexes, myenteric plexus activity, and perhaps even muscle-to-muscle transmission. The participation of vago-vagal reflexes is indicated not only by impairment of secondary peristalsis achieved by high vagal section in animals (13), but also by Andrew's findings in rats that the very passage of a contraction, without any bolus, is sufficient to release afferent impulses (14).

Secondary peristalsis may be defined as a wave stimulated by transient local distention of the esophagus, characterized by sequential phenomena of inhibition and excitation, and depending for its propagation neither on a swallowing center nor a moving bolus. Except for its method of stimulation and its apparent independence of a swallowing center, secondary peristalsis is thus identical in its manifestations to primary peristalsis. The question then arises: Are primary and secondary peristalsis really different? Is not primary peristalsis merely a peristaltic phenomenon precipitated when either a bolus or a contraction is passed from the pharynx into the esophagus? A number of observations made in moderately anesthetized animals would indicate that primary and secondary peristalsis differ in their modes of esophageal propagation $(1,4,13)$, but these observations are not unequivocally conclusive. The studies here reported and the erratic behavior of primary as well as secondary peristalsis suggest that the basic mechanisms underlying both types of esophageal peristalsis are essentially the same. 


\section{SUM MARY}

Esophageal peristalsis is traditionally separated into two types: primary, set off by swallowing and propagated by stimuli emanating from a medullary swallowing center; and secondary, initiated by esophageal distention and propagated by sequential short reflex arcs stimulated by the moving bolus. The studies underlying this concept of secondary peristalsis made use, however, of a moving bolus, a circumstance which makes it impossible to tell whether secondary peristalsis, once elicited, would similarly pass down the esophagus were no bolus present.

In our studies, secondary peristalsis was initiated by distending an esophageal balloon that was fixed, and by measuring the resultant motor and inhibitory phenomena above and below the balloon with the aid of manometric devices too small to affect motility. When the balloon was distended momentarily, the high resting pressure in the zone of the lower esophageal sphincter fell, and a pressure wave travelled down the esophagus. The total sequence was identical to that produced when primary peristalsis is initiated by a dry swallow. By varying the method of balloon inflation, motor and inhibitory phenomena identical to those produced by rapidly or slowly repeated swallows were obtained. Above the distending balloon, nonpropulsive contractions were the usual reactions to balloon distention. No evidence was obtained to indicate that a nervous swallowing center was controlling the secondary peristaltic phenomena elicited.

The propagation of secondary peristalsis thus does not require a moving bolus to stimulate sequential motor reflexes, nor does it appear to be dominated by a swallowing center. The orderly progress of secondary peristalsis presumably depends, therefore, on impulses released by the contraction itself and transmitted via vago-vagal, myenteric plexus, and perhaps intermuscular activity.

Primary and secondary peristalsis are remarkably similar in their manometric manifestations, their independence of an esophageal bolus, and their erratic incidence following apparently adequate stimuli. It may be questioned, therefore, whether any real difference between these two forms of esophageal motility exists and whether, indeed, a swallowing center has any influence over the esophageal phase of primary peristalsis.

\section{REFERENCES}

1. Hwang, $K$. Mechanism of transportation of the content of the esophagus. J. appl. Physiol. 1954, 6, 781.

2. Creamer, B., and Schlegel, J. Motor responses of the esophagus to distention. J. appl. Physiol. 1957, 10, 498.

3. Meltzer, S. J. On the causes of the orderly progress of the peristaltic movements in the oesophagus. Amer. J. Physiol. 1899, 2, 266.

4. Meltzer, S. J., and Auer, J. Vagus reflexes upon oesophagus and cardia. Brit. med. J. 1906, 2, 1806.

5. Fleshler, B., Hendrix, T. R., Kramer, $P$., and Ingelfinger, F. J. Resistance and reflex function of the lower esophageal sphincter. J. appl. Physiol. 1958, 12, 339.

6. Fyke, F. E., Jr., Code, C. F., and Schlegel, J. F. The gastroesophageal sphincter in healthy human beings. Gastroenterologia (Basel) 1956, 86, 135.

7. Kramer, P., and Ingelfinger, F. J. I. Motility of the human esophagus in control subjects and in patients with esophageal disorders. Amer. J. Med. 1949, 7, 168.

8. Kramer, P., and Ingelfinger, F. J. II. Cardiospasm, a generalized disorder of esophageal motility. Amer. J. Med. 1949, 7, 174.

9. Ingelfinger, F. J., and Abbott, W. O. Intubation studies of the human small intestine. XX. The diagnostic significance of motor disturbances. Amer. J. dig. Dis. 1940, 7, 468.

10. Butin, J. W., Olsen, A. M., Moersch, H. J., and Code, C. F. A study of esophageal pressures in normal persons and patients with cardiospasm. Gastroenterology 1953, 23, 278.

11. Burget, G. E., and Zeller, W. E. A study of the cardia in unanesthetized dogs. Proc. Soc. exp. Biol. (N. Y.) 1936, 34, 433.

12. Bosma, J. F. Deglutition: Pharyngeal stage. Physiol. Rev. 1957, 37, 275.

13. Ingelfinger, F. J. Esophageal motility. Physiol. Rev. 1958, 38, 533.

14. Andrew, B. L. The nervous control of the cervical oesophagus of the rat during swallowing. J. Physiol. 1956, 134, 729. 